\title{
Fault Forecasting of a Machining Center Tool Magazine Based on Health Assessment
}

\author{
Guofa Li, ${ }^{1,2}$ Yanbo Wang, ${ }^{1,2}$ Jialong He $\mathbb{D}^{1,2}$ Tianwei Hou, ${ }^{1,2}$ Le Du, ${ }^{1,2}$ and Zhenhua Hou ${ }^{1,2}$ \\ ${ }^{1}$ Key Laboratory of CNC Equipment Reliability, Ministry of Education, Jilin University, Changchun, Jilin, China \\ ${ }^{2}$ School of Mechanical and Aerospace Engineering, Jilin University, Changchun 130022, China \\ Correspondence should be addressed to Jialong He; hjl.star@163.com
}

Received 19 November 2019; Revised 5 February 2020; Accepted 2 March 2020; Published 9 April 2020

Academic Editor: Frederic Kratz

Copyright $\odot 2020$ Guofa Li et al. This is an open access article distributed under the Creative Commons Attribution License, which permits unrestricted use, distribution, and reproduction in any medium, provided the original work is properly cited.

\begin{abstract}
A tool magazine is one of the key functional components of machining centers with frequent faults. The reliability level of a tool magazine directly affects the reliability level of the machining center. After establishing a reliability test bench and a prognostic and health management system for a tool magazine, a novel fault-forecasting method for machining center tool magazines based on health assessment is proposed. First, the health status of each tool magazine subcomponent is determined using the grey clustering method. Second, the weight of each tool magazine subcomponent is determined using an entropy weight method. Third, the health status of the tool magazine is evaluated via fuzzy comprehensive evaluation. If the tool magazine exhibits an unhealthy status, then the subcomponent with the worst health status is selected for fault forecasting. In addition, standardized treatment, stationarity test, and differential processing are conducted separately on the raw performance indicator data of the worst subcomponent. Finally, the performance indicators of the worst subcomponent are forecasted with the constructed autoregressive moving average model. Using tool-falling failure as an example, the forecasted and experimental tool-pulling forces are compared and analyzed, and the prediction accuracy of the proposed method is verified.
\end{abstract}

\section{Introduction}

The numerical control (NC) machine tool is the material and technical basis of a modern manufacturing system; it is a "working machine" with high-tech content. At present, NC machine tools have made remarkable progress in terms of accuracy, speed, large-scale production, multiaxis linkage, and composite functions. However, their reliability level is one bottleneck that restricts their ability to reach the advanced international level. A tool magazine is one of the key functional components of $\mathrm{NC}$ machine tools with frequent faults that seriously affect its processing efficiency, resulting in considerable maintenance costs and downtime losses. Prognostic and health management (PHM) uses various algorithms and models to identify the mapping relationship between fault symptoms and causes, deduces the cause and location of faults, traces fault symptoms, predicts possible faults, points out the development trends and consequences of faults, predicts the residual life of components, and formulates the best maintenance and guarantee scheme. Therefore, PHM technology for tool magazines is helpful for improving their reliability, safety, and adaptability.

PHM technology has been gradually applied to engineering since the 1970s. The US military used PHM technology to build a health management system for F-35 Joint Strike Fighter [1]. Lee et al. [2] discussed the implementations of machine health management within a smart factory in terms of data connectivity, communications, cyber-physical systems, and virtual factories. Tsui et al. [3] provided a concise review of mainstream methods in aspects of the PHM framework with a focus on data-driven approaches. Lee et al. [4] provided a comprehensive review of PHM design for a rotary machinery system. Khan and Yairi [5] presented a systematic review of artificial intelligencebased PHM. He et al. [6] addressed the development and application of PHM technologies to a screw-tightening machine. Zheng et al. [7] designed a PHM system for NC machines to improve the reliability and availability of NC 
machine tools. Javed et al. [8] built a model with summation wavelet extreme learning machine, to perform tasks, such as the wear estimation of a cutting tool from a machining center and the long-term prediction of a bearing from a machining center.

In health assessment, Cui et al. [9] proposed a health assessment method for aircraft hydraulic systems based on fuzzy grey clustering, the analytic hierarchy process, and a combination weighting method. Li et al. [10] used weighted average method and fuzzy comprehensive evaluation method to improve conventional performance evaluation of health, safety, and environment. Dong et al. [11] proposed a health assessment method based on a combination weighting method and a cloud model for wind turbines. Zheng et al. [12] proposed a health assessment method based on a combination weighting method, a cloud model, and the analytic hierarchy process for wind turbines. Gul and Guneri [13] weighted the possibility and severity of parameters related to the hazards in an aluminum plate manufacturing plant by fuzzy analytic hierarchy process. Xu et al. [14] proposed a fuzzy theory-grey model-support vector machine approach for condition assessment of on-board integrated health management systems in manned spacecraft avionics. Sun et al. [15] integrated the merits of fuzzy set theory, fuzzy analytical hierarchical process, and modified weighted averaging combination and then proposed a novel evaluation model for transformer. Wu and Wu [16] assessed the health state of train control center by adopting the health index model of train control center based on fuzzy comprehensive evaluation. Wang et al. [17] proposed a multitype evidence synthesis method based on Dempster-Shafer evidence theory modified with an entropy weight method for the health assessment of a hydraulic system of a special vehicle. Liu et al. [18] evaluated the health status of overhead lines using the analytic hierarchy process and the technique for order of preference by similarity to an ideal solution. Wang et al. [19] proposed a Dempster-Shafer theory and information entropy weight-based granulation fusion method for determining the multisource health status information of electric motors. Yang et al. [20] proposed a radar health assessment method based on a combination of fuzzy comprehensive evaluation and cuckoo search-support vector regression. Li et al. [21] applied dynamic analytic hierarchy process method and the dynamic grey relation analysis method for risk assessment of tailings dam disaster status. Deng et al. [22] used two-direction locating accuracy, two-way repeated locating accuracy, reverse clearance error, and straightness to evaluate the health status of an NC machine tool feed system. Yin et al. [23] used a constraint covariance matrix adaptation evolution strategy algorithm to optimise the parameters of infinite irrelevance belief rulebased model and then evaluated the health status of the servo system of an NC machine tool. Chen et al. [24] used a single continuous hidden Markov model approach to evaluate the health status of machining centers. Classical health assessment methods include fuzzy comprehensive evaluation, grey clustering, and entropy weight method. The primary research objects are wind power and weapon equipment. In contrast with these research objects, the load of a tool magazine exhibits periodicity. For a tool magazine, the maximum pulling force of a tool shank can reach $5000 \sim 6000 \mathrm{~N}$, and the maximum tool-pulling force can reach $600 \sim 800 \mathrm{~N}$. Therefore, a new health assessment method is proposed to assess the health status of tool magazines.

In fault prediction, Aggoune et al. [25] proposed a fault detection method based on nonlinear autoregressive moving average (ARMA) with an exogenous input model and Hellinger distance for a distillation unit. Wu et al. [26] used a fractal autoregressive integrated moving average (ARIMA) model to predict the skip-over of a machinery. Jurjevcic et al. [27] used an ARIMA method for the early fault detection of a pulverised coal preparation system. Cai et al. [28] used an adaptive residual grey model and an improved ARMA model to predict gyroscope drift. Kim et al. [29] presented a forecasting approach based on generalised linear ARMA models and integer-valued generalised autoregressive conditional heteroscedastic models. Xiao et al. [30] used a multistep ARMA model to detect sensor and processed faults with good accuracy under different scenarios. Wang et al. [31] used an ARIMA model and a hidden Markov model to predict the reliability of a vertical roller mill. Aghdam and Cigeroglu [32] used a nonstationary functional series time-dependent ARMA model for forecasting tool nonstationary signals. At present, most fault-forecasting methods are based on the ARMA model and its variants. Compared with the ARMA model, the ARIMA model first performs differential processing on nonstationary time series and then establishes the ARMA model. The primary objects of fault forecasting are rotary parts, and the monitored signals are mostly vibration signals. Existing methods do not fully consider the physical meaning and degradation of the performance indicators of each subcomponent. Therefore, the generalization of these methods is weak, and their prediction accuracy is poor.

The current study builds a reliability test bench and a PHM system for tool magazines. The health status of each tool magazine subcomponent is evaluated using grey clustering and an entropy weight method. Meanwhile, the health status of a tool magazine is evaluated using a fuzzy comprehensive evaluation method. When a tool magazine exhibits an unhealthy status, fault forecasting is performed on the subcomponent with the worst health status. Using toolfalling failure as an example, we forecast the changing trend of the tool-pulling force.

\section{Relevant Theory and Methodology}

2.1. Grey Clustering Theory. Grey clustering theory is a method for integrating several performance indicators that cannot be directly classified into several grey categories by the whitening weight function of grey relational objects. For example, $m$ objects and $n$ performance indicators should be clustered, and $k$ different grey categories are present. $X_{i j}$ $(i=1,2, \ldots, m ; j=1,2, \ldots, n)$ is the evaluation value of the $i$-th clustering object on the $j$-th clustering index. Grey clustering refers to classifying the $i$-th object into the $s$-th $(s=\{1, \ldots, k\})$ grey category. The whitening weight function 
of the $s$-th grey category and the $j$-th performance indicator is recorded as $f_{j}^{s}(*)$. The whitening weight functions include whitening weight function of a typical measure, whitening weight function of a lower limit measure, whitening weight function of an upper limit measure, and whitening weight function of a moderate measure. $\beta_{i}^{s}$ is the grey clustering coefficient of the $i$-th clustering object relative to the $s$-th grey class:

$$
\begin{aligned}
\beta_{i}^{s} & =\sum_{j=1}^{n} f_{j}^{s}\left(X_{i j}\right) \cdot \omega_{j}^{s}, \\
\sum_{j=1}^{n} \omega_{j}^{s} & =1,
\end{aligned}
$$

where $\omega_{j}^{s}$ represents the weight of the $j$-th performance indicator and $s$-th grey class. The clustering coefficient vector of the $i$-th clustering object is shown in the following equation:

$$
\begin{aligned}
\beta_{i}=\left[\beta_{i}^{1}, \beta_{i}^{2}, \ldots \beta_{i}^{k}\right] & =\left[\sum_{j=1}^{n} f_{j}^{1}\left(X_{i j}\right) \cdot \omega_{j}^{1}, \ldots, \sum_{j=1}^{n} f_{j}^{k}\left(X_{i j}\right) \cdot \omega_{j}^{k}\right] \\
\max _{1 \leq s \leq k}\left\{\beta_{i}^{s}\right\} & =\beta_{i}^{s^{*}},
\end{aligned}
$$

where $\beta_{i}^{s^{*}}$ denotes that the $i$-th cluster object belongs to the $s^{*}$-th grey class.

2.2. Entropy Weight Method. Suppose $m$ objects and $n$ performance indicators exist. The original data matrix $X=\left(X_{i j}\right),\left(X_{i j} \geq 0, i=1,2, \ldots, m ; j=1,2, \ldots, n\right)$. Matrix $X$ is normalized to obtain matrix $P$ :

$$
\omega_{j}=\frac{\left(1-E_{j}\right)}{\left(n-\sum_{j=1}^{n} E_{j}\right)},
$$

where $\omega_{j}$ represents the weight of the $j$-th performance indicator. $E_{j}$ represents the entropy of the $j$-th performance indicator.

2.3. ARMA Model. ARMA is a stochastic time series model. As shown in equation (5), time series $\left\{X_{t}\right\}$ exhibits the characteristics of a stable, zero-mean, and normal distribution, and then it can be fitted by the ARMA model:

$$
X_{t}=\sum_{i=1}^{m} \varphi_{i} X_{t-i}-\sum_{j=1}^{n} \theta_{j} \tau_{t-j}+\tau_{t},
$$

where $m$ denotes the autoregressive order, $n$ denotes the moving average order, $\varphi_{i}$ is the autoregressive coefficient, $\theta_{j}$ is the moving average coefficient and sequence, and $\left\{\tau_{t}\right\}$ is white noise.

2.4. Determination of ARMA Model Order. The purpose of determining model order is to identify which model should be selected for fault prediction from the autoregressive (AR),
TABle 1: Judgment basis of model order.

\begin{tabular}{lccc}
\hline Model & AR $(m)$ & MA $(n)$ & ARMA $(m, n)$ \\
\hline ACF & Trailing & $n$ order truncation & Trailing \\
PACF & $m$ order truncation & Trailing & Trailing \\
\hline
\end{tabular}

moving average (MA), and ARMA models. In general, the order of a model is determined by observing the ACF and partial ACF (PACF) of a sequence. Table 1 shows the relationship between model order, ACF, and PACF.

The order of the ARMA model determined by ACF and PACF should be optimised. The general optimisation criteria include the Akaike information criterion and the Bayesian information criterion.

2.5. Model Solving and Testing. $m$ and $n$ are calculated in accordance with AIC and BIC. The results of $m$ and $n$ are expressed by $\hat{m}$ and $\hat{n}$. The unknown parameters in the model are calculated using SQP. General test methods include model goodness of fit, $F$, and residual tests. Theoretically, $\tau_{t}$ is a white Gaussian noise with a mean of zero. The residual of the preceding model is given by

$$
\widehat{\tau}_{t}=X_{t}-\sum_{i=1}^{m} \widehat{\varphi}_{i} X_{t-i}+\sum_{i=1}^{n} \widehat{\theta}_{i} \widehat{\tau}_{t-i}
$$

The residual test primarily tests the randomness of a sequence and determines whether it is white noise. If the residual sequence does not satisfy randomness, then the model should be improved. The hypothesis of the test is $\left\{H_{0}\right.$ : $\left.k \leq L, \rho_{k}=0 ; H_{1}: k \leq L, \rho_{k} \neq 0\right\}$. If $H_{0}$ holds, then $\chi^{2}$ approximates to the $\chi^{2}(L-r)$ distribution, where $r$ is the number of parameters of the ARMA model. For a given saliency level $\alpha, H_{0}$ is rejected when $x^{2}>x_{\alpha}^{2}(L-r)$; that is, $\tau_{t}$ is not white noise and the model test fails. When $x^{2} \leq x_{\alpha}^{2}(L-r), H_{0}$ is accepted; that is, $\tau_{t}$ is white noise, and the model test passes.

\section{Fault Forecast of a Tool Magazine Based on the ARMA Model}

3.1. Partition of a Tool Magazine and Determination of Performance Indicators. The research object in this work is the YP4024 tool magazine. We tracked a batch of tool magazines used by an enterprise. A reasonable division of subcomponents is the premise of PHM. In accordance with the principles of function sharing, function independence, and industry consensus, we divide the tool magazine into five subcomponents: the tool flywheel motor, the manipulator motor, the pneumatic device, the automatic tool change (ATC) system, and the cutter body and the tool case. In general, the tool flywheel motor and the manipulator motor are purchased parts. The pneumatic device, the ATC system, the cutter body, and the tool case have different functions. The ATC system primarily includes the transmission system of the manipulator and the manipulator. The failure mode is the manifestation of failure. After analyzing the collected faults, the typical failure modes of a tool magazine include toolfalling failure, jamming of tool failure, sensor failure, 


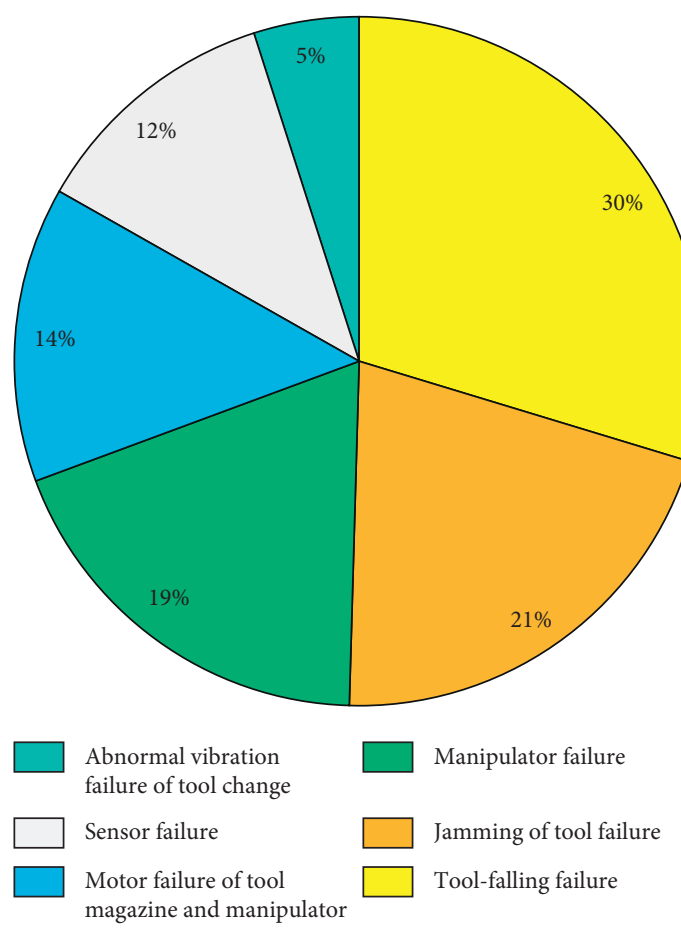

Figure 1: Proportion of the six types of failure modes.

manipulator failure, motor failure of the tool magazine and the manipulator, and the abnormal vibration failure of tool change. The frequency of the latter's occurrence is shown in Figure 1.

Fault tree analysis (FTA) is used to analyze the mechanism of every fault mode, and the relationship between fault mode and performance indicators is established from the level of fault mechanism. As shown in Figure 2, we determine the performance indicators of each subcomponent on the basis of the results of the aforementioned fault analysis.

\subsection{Fault Forecasting of a Tool Magazine Based on Health} Assessment. The fault forecasting process for tool magazines based on health assessment is shown in Figure 3. The specific steps are as follows:

Step 1: a tool magazine is divided into five different subcomponents, and the performance indicators of each subcomponent are determined.

Step 2: for every subcomponent, the weight of each performance indicator is determined using an entropy weight method, and the health status of each subcomponent is evaluated using the grey clustering method.

Step 3: the entropy weight method is used to determine the weight of each subcomponent. A fuzzy comprehensive evaluation method is used to determine the health status of the tool magazine.

Step 4: if the tool magazine exhibits an unhealthy status, we analyze failure modes related to the subcomponent with the worst health status and identify the most likely failure. Then, we determine the performance indicators corresponding to the most likely failure.
Step 5: the performance indicator data of the subcomponent with the worst health status are collected and standardized. Next, autocorrelation function (ACF) is used to test the stability of performance indicator data. If the data are nonstationary, then the data are smoothed by differential operation.

Step 6: the order of the ARMA model is determined on the basis of the ACF and PACF of the processed sequence. The order of the ARMA model is optimised through AIC and BIC. The unknown parameters in the model are calculated using sequence quadratic program (SQP). The ARMA model is tested via the residual test method. The failure of the tool magazine is forecasted on the basis of the established ARMA model.

\section{Results and Discussion}

4.1. Reliability Test Bench and PHM System of a Tool Magazine. The reliability test bench and PHM system of a tool magazine are established as shown in Figure 4. The reliability test bench includes a bracket, a tool magazine, various sensors, a counterweight block, and a virtual spindle. The counterweight block is used to replace the real tool. The PHM system consists of condition-monitoring, data management, health assessment, and fault-forecasting modules. The condition-monitoring module collects the performance indicator data. The data management module completes the storage and reading of the collected data. The health assessment module realizes the health assessment of the subcomponents and the tool magazine. The fault-forecasting module is used to forecast the failure of the subcomponent with the worst health status.

4.2. Health Assessment of a Tool Magazine. Table 2 shows the weights and normalized values of each performance indicator for several subcomponents of the tool magazine. Following the recommendation in [9], the normalized values are defined as healthy in $[0.85,0.9,-,-]$, subhealthy in $[0.6$, $0.7,0.8,0.85]$, degeneration in $[0.4,0.5,-, 0.6]$, deterioration in $[0.2,0.3,-, 0.4]$, and failure in $[-,-, 0.1,0.2]$. Table 3 provides the grey clustering coefficients and health status of each subcomponent of the tool magazine calculated on the basis of grey clustering theory. By substituting the data provided in Table 3 into the calculation formula of the entropy weight method, the weight value of each subcomponent of the tool magazine is determined as follows: $\omega=\{0.20,0.20,0.23,0.09,0.28\}$. The health status of the tool magazine can be obtained by using the fuzzy comprehensive evaluation method, as shown in Table 3. The evaluation result indicates that the health status of the tool magazine is subhealthy, and the cutter body and the tool case should be inspected after shutdown. After inspection, the tool-pulling force of No. 24 tool case is relatively small, and the throttle valve in the pneumatic device that controls the flow rate of the air intake of the cylinder is loose. On the basis of the aforementioned inspection and analysis, the evaluation result is consistent with the actual state. 


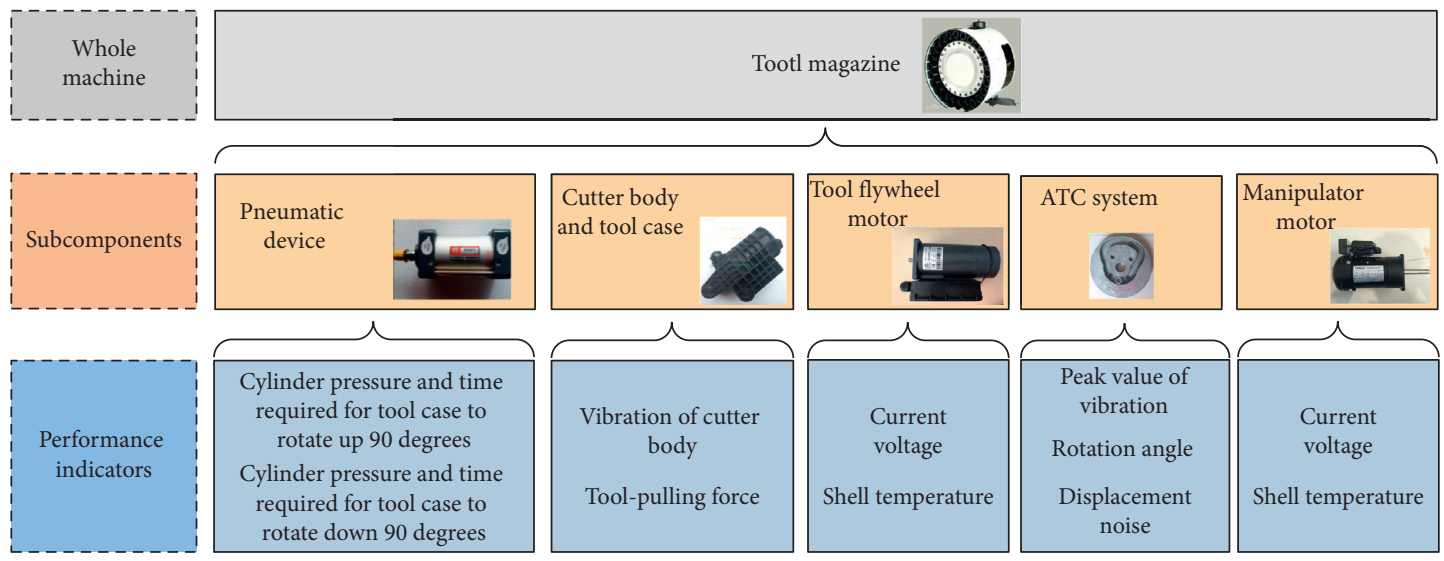

FIgURe 2: Performance indicators of every subcomponent.

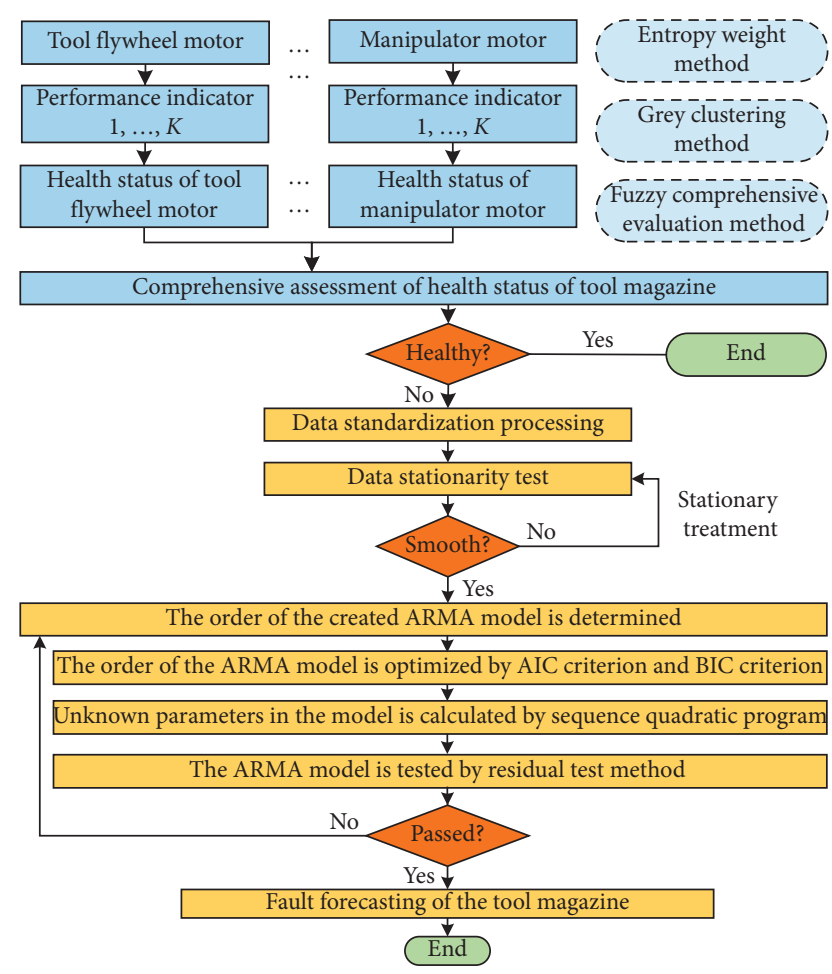

FIgURE 3: Fault forecasting of a tool magazine.

4.3. Fault Forecasting of a Tool Magazine. The health assessment result implies that the failure of the cutter body and the tool case should be forecasted. The failure rate of the cutter body is extremely low, and its performance indicator is vibration of cutter head. The tool case is a consumable part. Its performance indicator is tool-pulling force. The inspection of the cutter body and the tool case reveals that the locking force of No. 24 tool case is too small. Small locking force mainly causes the tool-falling failure. The toolfalling failure can be characterized by the tool-pulling force. We select the tool-falling failure as the most likely failure and predict the most important performance indicator, namely, the tool-pulling force. A total of 10000 tool changes are executed for No. 24 tool case. The tool-pulling force is measured every 100 tool changes. A total of 100 tool-pulling force values are measured. The specific change of the toolpulling force is shown in Figure 5.

The data in Figure 5 are standardized to calculate the ACF of the original data. As shown in Figure 6, $\widehat{\rho}_{L}$ does not converge to 0 with an increase in $L$; thus, the original sequence is nonstationary. Given the linear trend of the original data, the first-order differential processing of the original data is performed, as shown in Figure 7.

As indicated in Figure 7, the downward trend of the raw data has been eliminated and the fluctuation of the data has been basically stabilized. Figures 8 and 9 present the ACF and PACF values of the first-order differential sequence.

Referring to Figures 8 and 9, when $L$ is 0 , both PACF and ACF are 1 . As $L$ increases from 1 to 4 , the ACF decays rapidly, and the PACF basically exhibits an exponential decay. As $L$ increases from 5 to 20, ACF and PACF basically fluctuate slightly between $[-0.1,0.1]$. Because of the randomness of samples, ACF and PACF do not show theoretical truncation or tailing, but a slight oscillation. The ACF and PACF of the first-order differential sequence are a fourorder truncation and a tailing, respectively. Preliminary determination indicates that $m=0$ and $n=4$. The model is determined to be MA (4). On the basis of ACF and PACF, the order of the ARMA model is less than $4(m, n<4)$. AIC and BIC are calculated for the first-order differential sequence, as shown in Figures 10 and 11.

When $m=2$ and $n=4, \mathrm{AIC}$ and BIC are the smallest; thus, the ARMA model is ARMA $(2,4)$. Then, the parameter is estimated using SQP. The model parameters are $\widehat{\theta}=(-2.340,2.220,-0.862,0.153)$ and $\widehat{\varphi}=(-1.247,1.260)$. The residual model is tested on the basis of significance level $\alpha=0.05$. The test result is $H_{0}$; that is, the accuracy of the model meets the requirements. The 1st 90th tool-pulling force values are fed into the ARMA $(2,4)$ model to predict the 91th 100th tool-pulling force values. The parameter estimation of the ARMA model is essentially a problem of finding the minimum value of a constrained nonlinear multivariable function. The parameter estimation methods of ARMA $(2,4)$ model include interior point method, SQP, and active set method. As shown in Table 4, the predictions of the ARMA $(2,4)$ model using different parameter estimation algorithms are compared with the original data. 


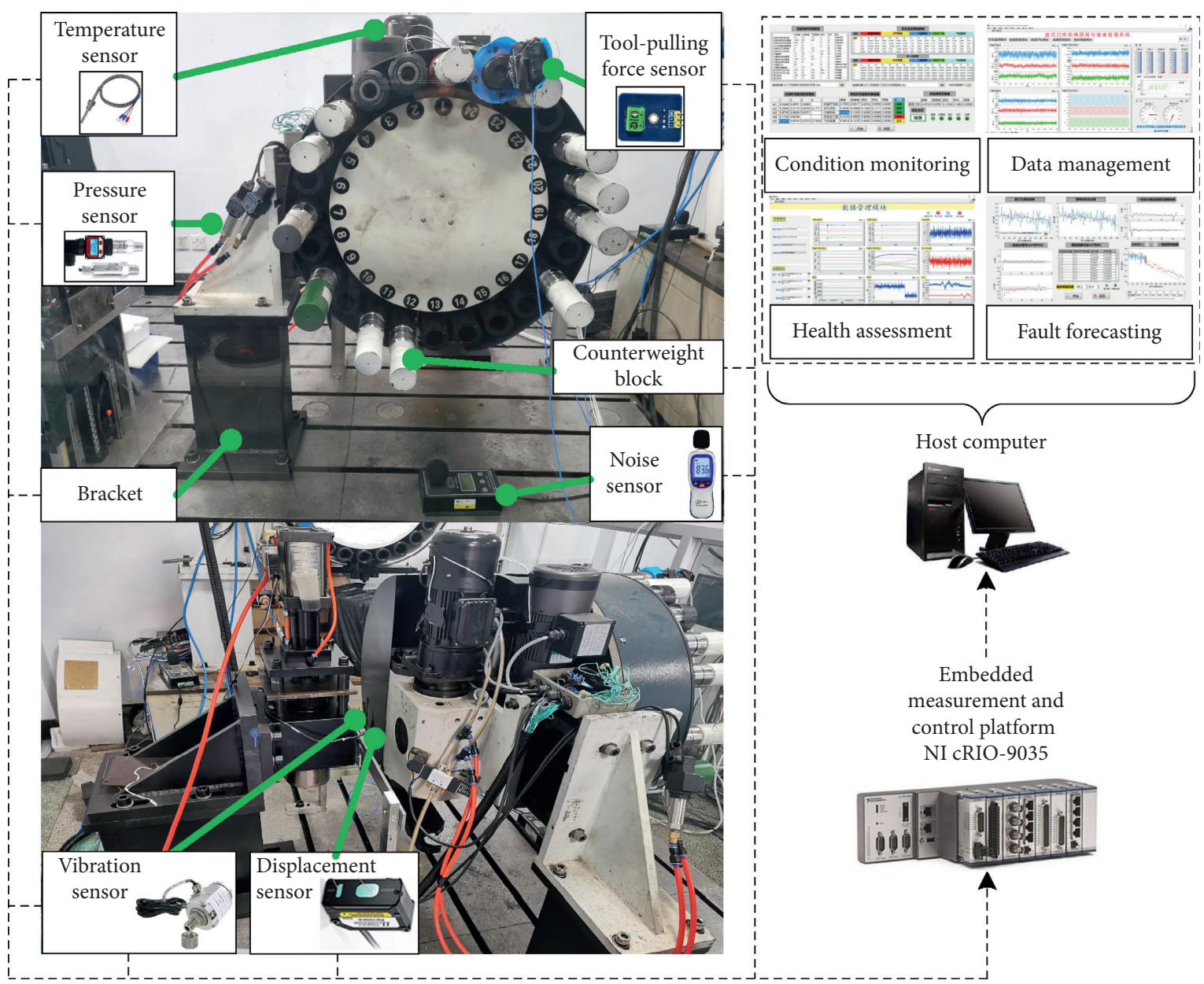

FIgURE 4: The reliability test bench and PHM system of a tool magazine.

TABLE 2: Weight and normalized value of every performance indicator for some subcomponents of a tool magazine.

\begin{tabular}{|c|c|c|c|c|c|c|}
\hline \multirow{2}{*}{$\begin{array}{l}\text { Subcomponent } \\
\text { Number }\end{array}$} & \multicolumn{3}{|c|}{ Manipulator motor } & \multicolumn{3}{|c|}{ Tool flywheel motor } \\
\hline & Current (A) & Voltage (V) & Shell temperature $\left({ }^{\circ} \mathrm{C}\right)$ & Current (A) & Voltage (V) & Shell temperature $\left({ }^{\circ} \mathrm{C}\right)$ \\
\hline 1 & 0.94 & 0.98 & 0.77 & 0.73 & 1.00 & 0.98 \\
\hline 2 & 0.79 & 0.98 & 0.82 & 0.76 & 0.99 & 0.88 \\
\hline 3 & 0.81 & 0.98 & 1.00 & 1.00 & 0.99 & 0.98 \\
\hline 4 & 0.99 & 0.98 & 0.82 & 0.69 & 0.99 & 1.00 \\
\hline 5 & 1.00 & 1.00 & 0.92 & 0.61 & 0.98 & 0.87 \\
\hline Weight $\omega_{j}$ & 0.94 & 0.01 & 0.05 & 0.88 & 0.08 & 0.04 \\
\hline
\end{tabular}

TABLE 3: Health status of every subcomponent and whole machine of a tool magazine.

\begin{tabular}{lcccccc}
\hline Subcomponent & Healthy & Subhealthy & Degeneration & Deteriorated & Fault & Health status \\
\hline Manipulator motor & 0.80 & 0.00 & 0.02 & 0.00 & 0.00 & Healthy \\
Tool flywheel motor & 0.88 & 0.12 & 0.00 & 0.00 & 0.00 & Healthy \\
Cutter body and tool case & 0.16 & 0.00 & 0.00 & 0.57 & 0.00 & Deteriorated \\
ATC system & 0.20 & 0.00 & 0.14 & 0.17 & 0.00 & Healthy \\
Pneumatic device & 0.00 & 0.13 & 0.40 & 0.00 & 0.00 & Degeneration \\
Tool magazine & 0.06 & 0.39 & 0.13 & 0.15 & 0.00 & Subhealthy \\
\hline
\end{tabular}

The original data fluctuated sharply. Each set of predictions fluctuates slowly. The mean absolute percentage error (MAPE) of the 10-step prediction of interior point method, SQP, and active set method is $2.34 \%, 2.26 \%$, and
$2.31 \%$, respectively. It can be seen that the $\operatorname{ARMA}(2,4)$ model with SQP has the highest prediction accuracy. The MAPE of the 10-step prediction of the ARMA $(2,4)$ model with SQP is within 3\%. In other words, the predicted value 


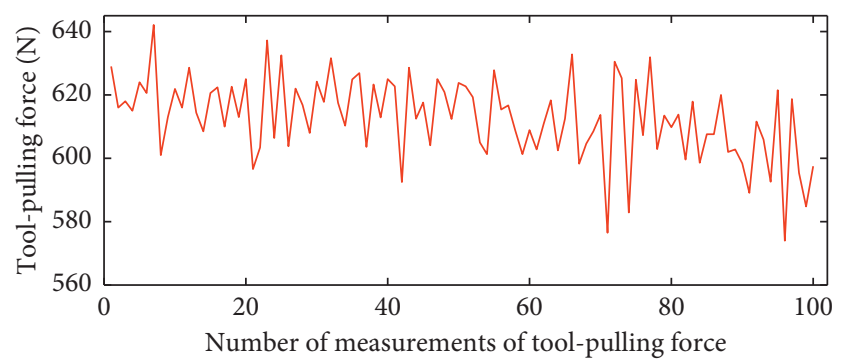

Figure 5: A schematic diagram of the change of tool-pulling force.

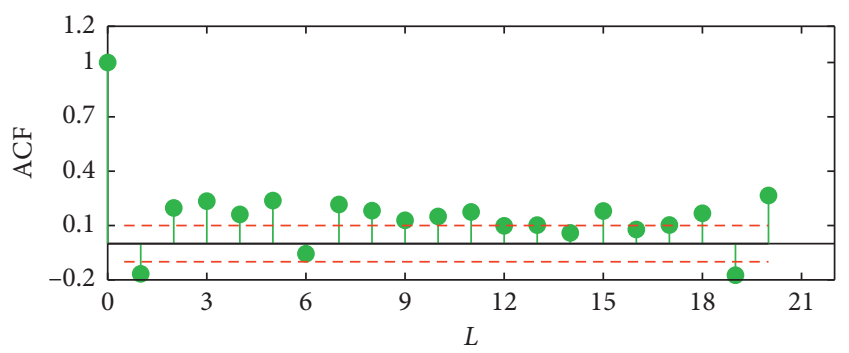

FIgURE 6: ACF of the raw data.

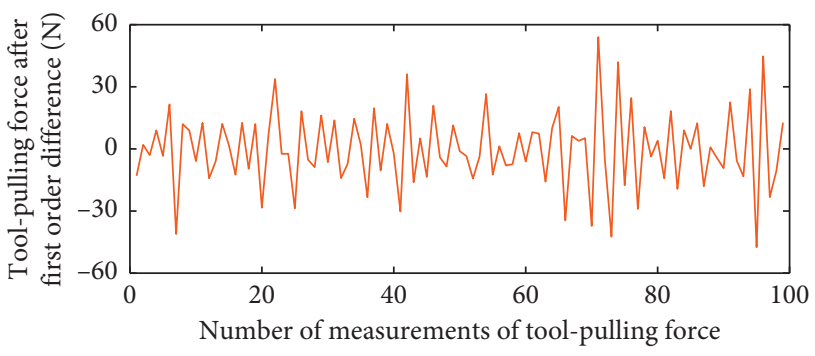

Figure 7: First-order difference sequence of raw data.

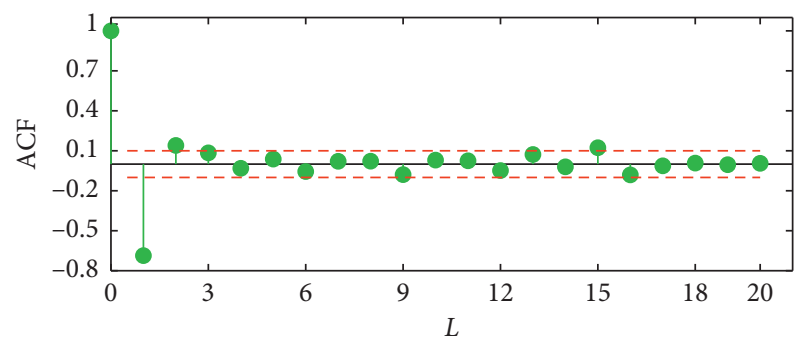

Figure 8: ACF of first-order differential sequence.

is likely to be 0.97 to 1.03 times the corresponding actual value. Firstly, it is generally believed that if the MAPE is less than $10 \%$, the prediction accuracy is considered to be high. Secondly, with the increase of the number of tool changes, the tool-pulling force decreased in a fluctuation way. Refer to Figure 7, the first-order difference sequence is not a constant value, but fluctuates basically in the interval $[-30 \mathrm{~N}, 30 \mathrm{~N}]$. It can be seen that the tool-pulling force has a certain randomness. Assume that the actual value of the tool-pulling force is $600 \mathrm{~N}$, its predicted value is likely to be between 580 and 620 . Finally, in engineering practice, the tool case is a consumable part. In order to

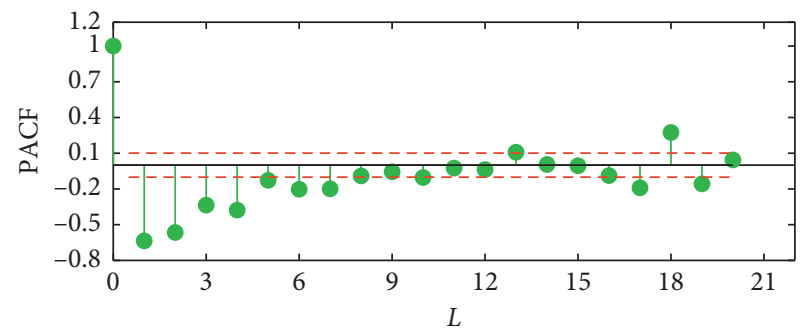

Figure 9: PACF of first-order differential sequence.

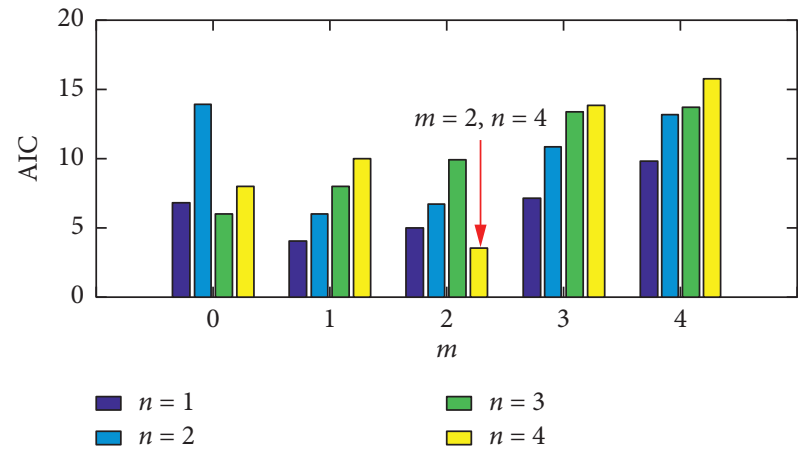

Figure 10: AIC for different $m$ and $n$.

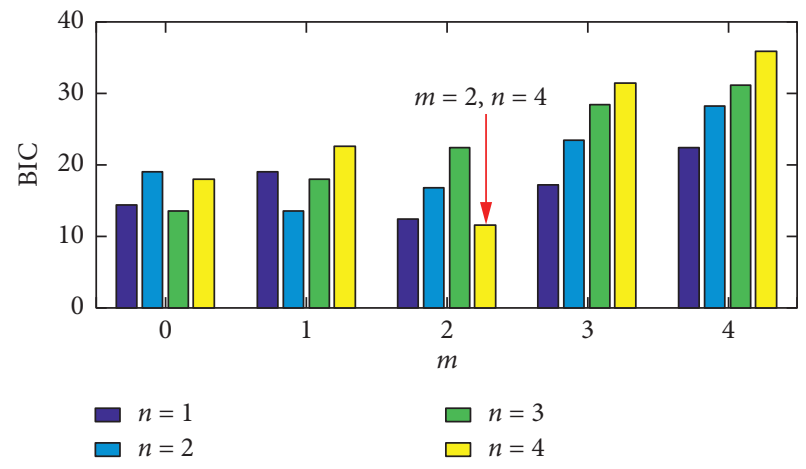

Figure 11: BIC for different $m$ and $n$.

replace the tool case in time, the failure threshold is often set to about 1.1 times the tool-pulling force when the toolfalling failure falls. Therefore, the constructed ARMA model is suitable for predicting tool-pulling forces.

As shown in Figure 12, the 1st 100th tool-pulling force values are fed into the ARMA $(2,4)$ model to predict the 101 th 515th tool-pulling force values. In the short term, for 101 th 110 th tool-pulling force values, the tool-pulling force decreased in a fluctuation way. Its fluctuation became smaller and smaller. The ARMA $(2,4)$ model has high prediction accuracy and retains small fluctuations. Given that the locking force of the tool case is less than $500 \mathrm{~N}$, toolfalling failure may occur. Thus, $500 \mathrm{~N}$ is used as the failure threshold of tool-falling failure. Obviously, the tool-falling failure will not happen shortly. In the long run, for 111th 515th tool-pulling force values, the tool-pulling force decreases linearly. Due to the nonlinearity and nonstationarity of the degradation process of the internal parts 
TABLE 4: Comparison between the original and the predicted values of the ARMA $(2,4)$ model using different parameter estimation algorithms.

\begin{tabular}{lccccccccc}
\hline Number & Actual value & Interior point & SQP & Active set & Number & Actual value & Interior point & SQP & Active set \\
\hline 91 & 589.1 & 610.0 & 608.3 & 610.0 & 96 & 574.0 & 605.9 & 604.0 & 605.9 \\
92 & 611.6 & 607.9 & 605.0 & 607.9 & 97 & 618.7 & 604.6 & 603.9 & 604.6 \\
93 & 605.9 & 604.8 & 604.4 & 604.8 & 98 & 595.4 & 605.3 & 603.9 & 605.3 \\
94 & 592.6 & 606.6 & 604.7 & 606.6 & 99 & 584.8 & 604.3 & 603.6 & 604.3 \\
95 & 621.5 & 604.8 & 604.6 & 604.8 & 100 & 597.5 & 604.8 & 603.3 & 604.8 \\
\hline
\end{tabular}

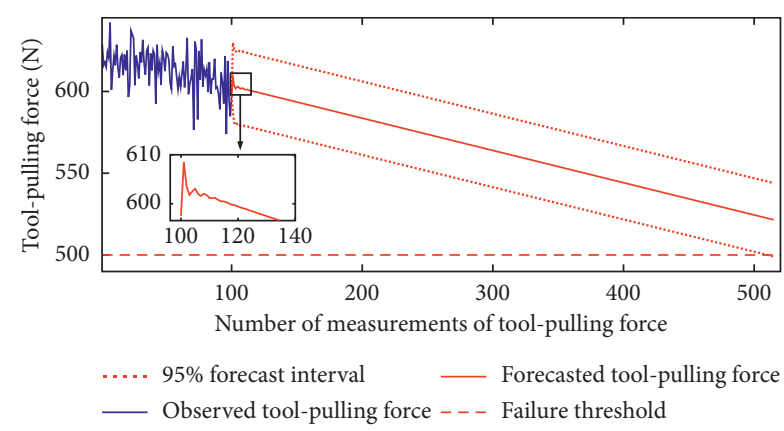

FIGURE 12: Change of forecasted tool-pulling force.

of tool case and the mutual coupling between the parts, the attenuation process of tool-pulling force is actually nonlinear and nonstationary. Therefore, the deviation between the linear attenuation process predicted by the constructed static ARMA $(2,4)$ model and the actual nonlinear attenuation process is large. Long-term prediction is inaccurate, but it is helpful to predict tool-falling failure. After 51000 tool changes, the forecasted interval of the tool-pulling force is [ $500 \mathrm{~N}, 545 \mathrm{~N}$ ]. Tool-falling failure may occur after 51000 tool changes.

\section{Conclusions}

The typical failure modes of a tool magazine are analyzed on the basis of a large number of failures. After establishing a reliability test bench and a PHM system for the tool magazine, a novel fault-forecasting method for the machining center's tool magazine based on health assessment is proposed. This research exhibits theoretical significance and practical application value. Simultaneously, it can be applied to other key functional components of the machining center and the entire machine, promoting the intelligentisation of the machining center. The major conclusions of this study are as follows:

(1) Applying the principles of function sharing, function independence, and industry consensus, the tool magazine is divided into five parts: the tool flywheel motor, the manipulator motor, the pneumatic device, the ATC system, and the cutter body and the tool case. We determine the performance indicators of each subcomponent from the FTA results. The PHM system of the tool magazine is established accordingly.

(2) A health assessment method based on grey clustering, entropy weight method, and fuzzy comprehensive evaluation method is proposed. The weight of each subcomponent's performance indicator is determined using the entropy weight method, and the health status of each subcomponent is evaluated via grey clustering. The health status of the manipulator motor, the tool flywheel motor, the cutter body and the tool case, the ATC system, and the pneumatic device are as follows: healthy, healthy, deteriorated, healthy, and degraded. The fuzzy comprehensive evaluation method is used to obtain the health status of the tool magazine as subhealthy.

(3) A fault prediction method based on the ARMA model is proposed. The evaluation result of the health status indicates that failure prediction should be performed on the No. 24 tool case. A total of 10000 tool changes are implemented, and the ARMA $(2,4)$ model is established. The model parameters $\widehat{\theta}=(-2.340,2.220,-0.862,0.153)$ and $\widehat{\varphi}=(-1.247,1.260)$. The trend of the tool-pulling force is predicted on the basis of the model, and the original data are used to verify the prediction result. The result shows that the mean absolute percentage error of 10 -step prediction is within $3 \%$. The constructed ARMA model is suitable for predicting tool-pulling forces.

\section{Data Availability}

The data used to support the findings of this study are included within the supplementary information file.

\section{Conflicts of Interest}

The authors declare that there are no conflicts of interest regarding the publication of this paper.

\section{Acknowledgments}

This work was supported by the State Key Science and Technology Program of China (Grant no. 2019ZX04012001), National Natural Science Foundation of China (51905209), Industrial Technology Research and Development Project of Jilin Province Development and Reform Commission, China (2019C040-2), Young and Middle-aged Scientific and Technological Innovation leaders and Team Projects in Jilin Province, China (20190101015JH), and Program for JLU Science and Technology Innovative Research Team (JLUSTIRT). 


\section{Supplementary Materials}

Table 1: original value of every performance indicator for every subcomponent of a tool magazine. Table 2: the 100 original tool-pulling force values. (Supplementary Materials)

\section{References}

[1] G.-Y. Kim, J.-K. Hwang, Y.-K. Im, and S.-W. Ha, "Roadmap configuration for technical elements acquisition of military fixed wing aircraft parts PHM and verification of parts selection phase," Journal of the Korean Society for Aeronautical \& Space Sciences, vol. 47, no. 9, pp. 665-677, 2019.

[2] G.-Y. Lee, M. Kim, Y.-J. Quan et al., "Machine health management in smart factory: a review," Journal of $\mathrm{Me}$ chanical Science and Technology, vol. 32, no. 3, pp. 987-1009, 2018.

[3] K. L. Tsui, N. Chen, Q. Zhou, Y. Z. Hai, and W. B. Wang, "Prognostics and health management: a review on data driven approaches," Mathematical Problems in Engineering, vol. 2015, Article ID 793161, 17 pages, 2015.

[4] J. Lee, F. J. Wu, W. Y. Zhao, M. Ghaffari, L. X. Liao, and D. Siegel, "Prognostics and health management design for rotary machinery systems-Reviews, methodology and applications," Mechanical Systems and Signal Processing, vol. 42, no. 1-2, pp. 314-334, 2014.

[5] S. Khan and T. Yairi, "A review on the application of deep learning in system health management," Mechanical Systems and Signal Processing, vol. 107, pp. 241-265, 2018.

[6] C. He, J. M. Li, and G. Vachtsevanos, "Prognostics and health management of an automated machining process," Mathematical Problems in Engineering, vol. 2015, Article ID 651841, 10 pages, 2015.

[7] R. Zheng, H. W. Sun, and Y. Z. Zhang, "Research on prognostics and health management technology of numerical control equipment," in Health Monitoring of Structural and Biological Systems 2014, T. Kundu, Ed., Spie-Int Soc Optical Engineering, Bellingham, WA, USA, 2014.

[8] K. Javed, R. Gouriveau, N. Zerhouni, and P. Nectoux, "Enabling health monitoring approach based on vibration data for accurate prognostics," IEEE Transactions on Industrial Electronics, vol. 62, no. 1, pp. 647-656, 2015.

[9] J. Cui, Z. Lin, R. Lv, L. Jiang, and Y. Qi, "Comprehensive assessment method of aircraft health status based on fuzzy gray clustering and combination weighting," Acta Aeronautica et Astronautica Sinica, vol. 35, no. 3, pp. 764-772, 2014.

[10] W. Li, W. Liang, L. Zhang, and Q. Tang, "Performance assessment system of health, safety and environment based on experts' weights and fuzzy comprehensive evaluation," Journal of Loss Prevention in the Process Industries, vol. 35, pp. 95-103, 2015.

[11] X. Dong, X. Zhang, K. Zheng, and S. Wang, "Health status assessment of wind turbine based on combination weighting and cloud model," Acta Energiae Solaris Sinica, vol. 39, no. 8, pp. 2139-2146, 2018.

[12] K. Zheng, L. Han, S. Guo, Z. Wang, X. Zhang, and X. Dong, "Fuzzy synthetic condition assessment of wind turbine based on combination weighting and cloud model," Journal of Intelligent \& Fuzzy Systems, vol. 32, no. 6, pp. 4563-4572, 2017.

[13] M. Gul and A. F. Guneri, "A fuzzy multi criteria risk assessment based on decision matrix technique: a case study for aluminum industry," Journal of Loss Prevention in the Process Industries, vol. 40, pp. 89-100, 2016.
[14] J. Xu, Z. Meng, and L. Xu, "Integrated system health management-based fuzzy on-board condition prediction for manned spacecraft avionics," Quality and Reliability Engineering International, vol. 32, no. 1, pp. 153-165, 2016.

[15] L. Sun, Z. Ma, Y. Shang, Y. Liu, H. Yuan, and G. Wu, "Research on multi-attribute decision-making in condition evaluation for power transformer using fuzzy AHP and modified weighted averaging combination," IET Generation, Transmission \& Distribution, vol. 10, no. 15, pp. 3855-3864, 2016.

[16] X. C. Wu and J. Wu, "Research on life prediction of train control center based on random and fuzzy theory," International Journal of Security and Its Applications, vol. 10, no. 1, pp. 375-384, 2016.

[17] L. Wang, W.-m. Lv, and Y.-c. Jin, "A combination assessment method based on multi-types of evidence," Control and Decision, vol. 29, no. 11, pp. 1973-1978, 2014.

[18] Y. Liu, J. Xv, H. Yuan, J. Lv, and Z. Ma, "Health assessment and prediction of overhead line based on health index," IEEE Transactions on Industrial Electronics, vol. 66, no. 7, pp. 5546-5557, 2019.

[19] L. Z. Wang, Y. S. Sun, Y. D. He, X. J. Zhao, W. H. Fan, and X. H. Wang, "Motor health status prediction method based on information from multi-sensor and multi-feature parameters," Journal of Nondestructive Evaluation, vol. 38, no. 2, p. 14, 2019.

[20] Y. F. Yang, M. H. Zhang, and Y. W. Dai, "A Fuzzy Comprehensive CS-SVR Model-based health status evaluation of radar," PLoS One, vol. 14, no. 3, Article ID e0213833, 2019.

[21] W. Li, Y. C. Ye, N. Y. Hu, X. H. Wang, and Q. H. Wang, "Realtime warning and risk assessment of tailings dam disaster status based on dynamic hierarchy-grey relation analysis," Complexity, vol. 2019, Article ID 5873420, 14 pages, 2019.

[22] C. Deng, Y. Wang, J. Wu, S. Xia, and Z. Tao, "Performance degradation modelling and health state evaluation for mechanical and electrical equipment," Computer Integrated Manufacturing Systems, vol. 24, no. 9, pp. 2279-2287, 2018.

[23] X. Yin, B. Zhang, Z. Zhou, X. Han, Z. Wang, and G. Hu, “A new health estimation model for CNC machine tool based on infinite irrelevance and belief rule base," Microelectronics Reliability, vol. 84, pp. 187-196, 2018.

[24] L. Chen, L. Tieying, and L. Hongmei, "Online milling tool condition monitoring with a single continuous hidden Markov models approach," Journal Of Vibroengineering, vol. 16, no. 5, pp. 2448-2457, 2014.

[25] L. Aggoune, Y. Chetouani, and H. Radjeai, "Change detection in a distillation column using non-linear auto-regressive moving average with exogenous input model and Hellinger distance," IET Science, Measurement \& Technology, vol. 10, no. 1, pp. 10-17, 2016.

[26] B. Wu, Y. D. Gao, S. L. Feng, and T. Chanwimalueang, "Sparse optimistic based on lasso-LSQR and minimum entropy deconvolution with FARIMA for the remaining useful life prediction of machinery," Entropy, vol. 20, no. 10, p. 16, 2018.

[27] B. Jurjevcic, A. Senegacnik, and I. Kustrin, "A surveillance of direct-firing system for pulverized-coal using statistically treated signals from intrusive electrostatic sensors," Strojniški vestnik-Journal of Mechanical Engineering, vol. 63, no. 4, pp. 265-274, 2017.

[28] X. W. Cai, C. X. Zhang, S. Gao, L. Wang, and X. M. Li, “A novel method based on a high-dynamic hybrid forecasting model for fiber optic gyroscope drift," Sensors and Materials, vol. 29, no. 1, pp. 1-13, 2017. 
[29] J. Y. Kim, H. Y. Kim, D. Park, and Y. Chung, "Modelling of fault in RPM using the GLARMA and INGARCH model," Electronics Letters, vol. 54, no. 5, p. 297, 2018.

[30] H. Xiao, D. Huang, Y. Pan, Y. Liu, and K. Song, "Fault diagnosis and prognosis of wastewater processes with incomplete data by the auto-associative neural networks and ARMA model," Chemometrics and Intelligent Laboratory Systems, vol. 161, pp. 96-107, 2017.

[31] Q. Wang, Y. L. Fang, Z. Zhou, J. Zuo, Q. L. Xiao, and S. J. Zhou, "Reliability assessment of the vertical roller mill based on ARIMA and multi-observation HMM," Cogent Engineering, vol. 4, no. 1, p. 16, 2017.

[32] B. H. Aghdam and E. Cigeroglu, "Vibration-based tool wear estimation by using non-stationary Functional Series TARMA (FS-TARMA) models," The International Journal of Advanced Manufacturing Technology, vol. 93, no. 1-4, pp. 1431-1442, 2017. 